\title{
Motivation and Belief in the Paranormal in a Remote Viewing Task
}

\author{
Eugene Subbotsky and Adrian Ryan
}

Psychology Department, Lancaster University, Lancaster LA1 4YF, UK

\begin{abstract}
In two experiments, the hypothesis was tested that participants' ability to remotely access the picture they would be shown in the nearest $15 \mathrm{~min}$. depends of the magnitude of the reward. In Experiment 1, in the no-extra reward condition participants received a regular pay of $£ 4$ for participation independently of the success of their attempt to access the target picture, whereas in the extra-reward condition participants who successfully identified the picture were paid an additional sum of $£ 80$ on top of their regular pay. Results indicated that in the no-extra reward condition participants scored positively and significantly above zero, whereas in the extra reward condition the remote viewing score was negative and not significantly different from zero. In Experiment 2, the reward in the extra reward condition was decreased form $£ 80$ to $£ 8$ for a successful attempt. In both conditions of this experiment participants scored around zero. In both experiments, participants' performance was at their best in the first 25 trials, and deteriorated during the subsequent 25 trials. In Experiment 1 the drop in performance was statistically significant. The study did not support the hypothesis that an additional material reward improves ESP performance. Nevertheless, the results partially support the ESP based experimenter's expectancy effect.
\end{abstract}

Keywords: Belief in paranormal, ESP, experimenter's expectancy effect, motivation, remote viewing.

\section{PROBLEM}

Rex Stanford's "Psi-mediated instrumental response" (PMIR) model [1] predicts that in everyday life people's $\mathrm{ESP}^{1}$ performance is positively affected by their needs to achieve goals. However, the authors did not manipulate need directly, only indirectly. For example, in one study participants had an opportunity to "help" a stranger to win an attractive activity against the unattractive one by reacting in the "right manner" to one of the 10 key words in a free association test. The results seem to indeed show that participants subconsciously reacted to the key words more often than chance. The authors interpreted the result as favouring the PMIR model: participants were able to access the key words via ESP and react to these words in the right manner because they had a subconsciously activated need to help the stranger.

This is a shaky assumption though: in psychology, a strong argument exists against the assumption that a human person has an "inbuilt" tendency to help a stranger in need even if his or her helping behaviour is directly challenged, to say nothing of the situation when the direct challenge is absent [2].

Drawing on this problem with the PMIR model, Eysenck \& Sargent commented "Obviously, if we had studies, which clearly varied the strength of some salient need, and showed

*Address correspondence to this author to the Psychology Department, Lancaster University, Lancaster LA1 4YF, UK;

E-mail: e.subbotsky@lancaster.ac.uk

${ }^{1}$ Extrasensory perception (ESP) is the ability to access information through time or space by means unknown to modern science. that ESP performance was related to the strength of that need, we would have better support for the PMIR theory. A word of warning, however: psychologists... are well aware that, under conditions of excessive need, performance in tests of skill tends to deteriorate... So the relation between need strength and ESP strength may not be a simple one... More studies of this kind are badly needed." [3, pp.123-124].

Following the above suggestion, Experiment 1of this study aimed at exploring the direct effect of motivation (need) on performance on an ESP task. ${ }^{2}$

\section{EXPERIMENT 1}

Method

\section{Participants}

Participants were 50 graduates and undergraduates at Lancaster University, UK, 23 males and 27 females, age range 18 to $29, \mathrm{M}=21.4, \mathrm{SD}=2.71$. None of the participants had taken part in any psi research before. Each participant completed one trial and the study comprised a total of 50 trials. Twenty-five participants were involved in each of the 2 conditions: extra reward (ER) and no extra reward (NER).

\section{Materials}

A Vaio laptop computer with the set of 300 photographs selected by [5] and a software program developed by the second author, a G-4 Mac laptop on which the instruction to participants was played, sheets of A4 plain paper and pencils

${ }^{2}$ This experiment has been presented at the 33rd International Conference of the Society for Psychical Research, Nottingham, U.K., [4] 
were used as materials. The sheep-goat ( $\mathrm{S} \& \mathrm{G}$ ) scale, which measured the degree of participants' belief in the ESP phenomena (such as psycho-kinesis or telepathy) [6] was also employed.

\section{Procedure}

The room was prepared by ensuring sufficient supply of pens and unlined white paper. The participant was asked whether they had something that would disturb them in a cognitive task. For example, if they were hungry, tired, mentally distracted or pressed for time, they may not perform at their best in such a task. If any of these apply at the current time, the session was aborted.

The experimenter then determined whether this participant is in the ER or NER condition group. This was achieved by a random method that ensures equal numbers in each group. If the participant was in the ER group, they were promised a payment of extra $£ 80$ for success, on top of their regular pay of $£ 4$ for their time. It was explained that as the experimenter would judge the viewer's drawings, the task will be a joint effort, but that once the judge's ratings are entered into the computer, they couldn't be altered.

The experimenter then played a narrative recorded on $\mathrm{CD}$, through a laptop computer, which gave a background of the remote viewing technique, explained the procedure, and took the participant through an exercise to clear their mind of any mental activity they were currently experiencing. The narrative explained to the participant that the task required them to mentally access and draw on a sheet of paper the target photograph, which will be later shown to them. The target photograph will be an outdoor scene, which will not contain any of the following: people, transportation devices (e.g., boats, cars, etc.), and small human artefacts (e.g., furniture, tools, toys, etc.).

Next, the experimenter asked the participant to write their name, date, and time on the upper right portion of the page and number the page as page one.

The experimenter then began the remote viewing by giving a stylised tasking to the participant: "There is a photograph that requires a description. Access to that photograph is through the trigger word 'Target.' When you are ready, place your pen on the page."

The experimenter would then say "Target". When the participant hears "Target", they will write the word Target and then immediately scribble a brief sketch and/or words of their first impression. If they hesitate, the experimenter would say, "Break". The participant writes "Break" on the page also. If they register a quick response the experimenter would also say, "Break" to momentarily end the remote viewing. The experimenter would then re-start the session by saying "Target".

The number of target/break couplets was not fixed. The time between break and the next trigger word target was also not fixed, but typically was $10-30 \mathrm{sec}$. In this time the experimenter engaged the participant with small talk, i.e., in some kind of conversation other than anything with regard to the session. This was to stop the participant from pondering what the target could be or from analysing their responses so far. If there was a long break (e.g., the participant needed a

bathroom break) or the break lasted many minutes with small talk, the experimenter re-tasked the participant as above.

Part of the experimenter's responsibility was to make sure that the responses made it to the paper. Often a novice participant would describe in words and gestures rather than writing. The experimenter may interrupt and ask that those words be added to the paper. The experimenter may also seek clarification, i.e., ask the participant if there are any words associated with the sketch. At no time did the experimenter ask leading questions or provided interpretation of what the participant had written or drawn.

Finally, the experimenter changed the tasking; "A summary of your impressions is required. Access to that summary is through the trigger word 'Target"'. Then the last stimulus was provided and the participant was encouraged to summarise their fragmented impressions and to label their sketches. After the summary the experimenter would say "Break" one last time and the participant was instructed to write EOS after their last entry to indicate End of Session. The remote viewing session lasted between 5 and 10 minutes.

The participants then completed a short questionnaire. Whilst they did so, the experimenter started a computer program, which displayed a randomly selected set of five photographs. The experimenter then scored the similarity between the participant's drawing and each photograph between 0 and 100 and entered this rating into the program. It was important that the participant sees only the target photograph, so the experimenter did the scoring with the computer screen being not visible to participants. When all of the ratings were entered, the computer program would randomly select one of the photos as the target, which would then be displayed to the participant.The procedure lasted no more than 30 minutes in total.

To summarize, the procedure consisted of 5 steps:

- Brief introduction.

- Playing CD with the detailed description of the experiment.

- Participant's drawing pictures.

- Participant's summarizing the results.

- The experimenter's scoring the participant's pictures.

- Displaying the target picture to the participant.

\section{Scoring}

On each session trial a $\mathrm{Z}$ score was computed, according to the formula $Z=$ (Target rating - Mean of trial ratings) $/ \mathrm{SD}$ of trial ratings, where Target rating was the rating $(0-100)$ assigned in the session to the actual target, Mean of trial ratings was average of all 5 ratings assigned in that trial.

The S \& G scale was scored as in Thalbourne \& Dean [6], by summing up the scores obtained through a visual analogue scale between "complete belief" and "complete disbelief" in a certain effect. 


\section{Design}

The between variable was condition (2: ER versus NER), and a within variable was test (2: the remote viewing test and the $S$ \& $\mathrm{G}$ questionnaire test). The dependent variables were scores on each of the tests.

\section{Expectations}

We expected that participants in the ER condition would score better than those in the NER condition. We also expected that the degree of participants' belief in the ESP would correlate positively with success on this task.

\section{Results}

In the NER condition, mean $\mathrm{Z}=0.41, \mathrm{SD}=0.72$. Onesample $t$-test indicated that in this condition the deviation of mean $\mathrm{Z}$ from zero is significant, with $\mathrm{t}=2.89, \mathrm{p}=0.008$ (two tailed). In the ER condition, mean $\mathrm{Z}=-.083, \mathrm{SD}=0.93$. According to one-sample $\mathrm{t}$-test, in this condition mean $\mathrm{Z}$ was not significantly different from zero, with $\mathrm{t}=-0.447$, $\mathrm{p}=0.659$ (two tailed).

A two-way ANOVA for condition indicated that in the NER condition participants scored significantly higher than in the ER condition, $\mathrm{F}(1,48)=4.51, \mathrm{p}=0.039$ (two tailed), $\eta 2=0.09$.

Correlations between the $\mathrm{S} \& \mathrm{G}$ scores on the first two scales (that assess the participants' belief in ESP) and the trial $\mathrm{Z}$ scores were not significant, with $\mathrm{r}=0.17, \mathrm{p}=0.11$ (2-tailed) in the ER condition, and $r=-0.20, p=0.41$ (2-tailed) in the NER condition.
Overall success on the task declined significantly with the time of testing. In the first half of testing ( 25 trials), mean $\mathrm{Z}=0.42, \mathrm{SD}=0.7$, and in the second (25 trials) mean $\mathrm{Z}=-0.09, \mathrm{SD}=0.94$. A one-way ANOVA for time of testing (2) indicated a significant difference, $F(1,48)=4.79$, $\mathrm{p}=0.033, \eta 2=0.09$. Fig. (1) illustrates the decline effect.

\section{Discussion}

In the NER condition, participants showed a significant ESP effect.

In contrast, in the ER condition no ESP effect was detected. The hypothesis that participants who were promised a reward for success would score better than those in the no extra reward group was not supported.

The hypothesis that the degree of belief in the ESP would correlate positively with success was not supported either, at least as far as the first two S \& G scales are concerned.

Altogether, the results of this experiment did not favour the assumption that the increased need to have an ESP effect increases the chance of succeeding on the ESP tasks.

One way of explaining these results is through participants' inability to access the target picture in the ER condition, due to their excessive level of anxiety. Indeed, the Yerkes-Dodson "optimum of motivation law" contends that optimal task performance occurs at an intermediate level of arousal, with relatively poorer performance occurs at both lower and higher arousal levels, leading to an inverted $U$ relation between arousal and performance [7]. In concordance with this law, in the ER condition the increased

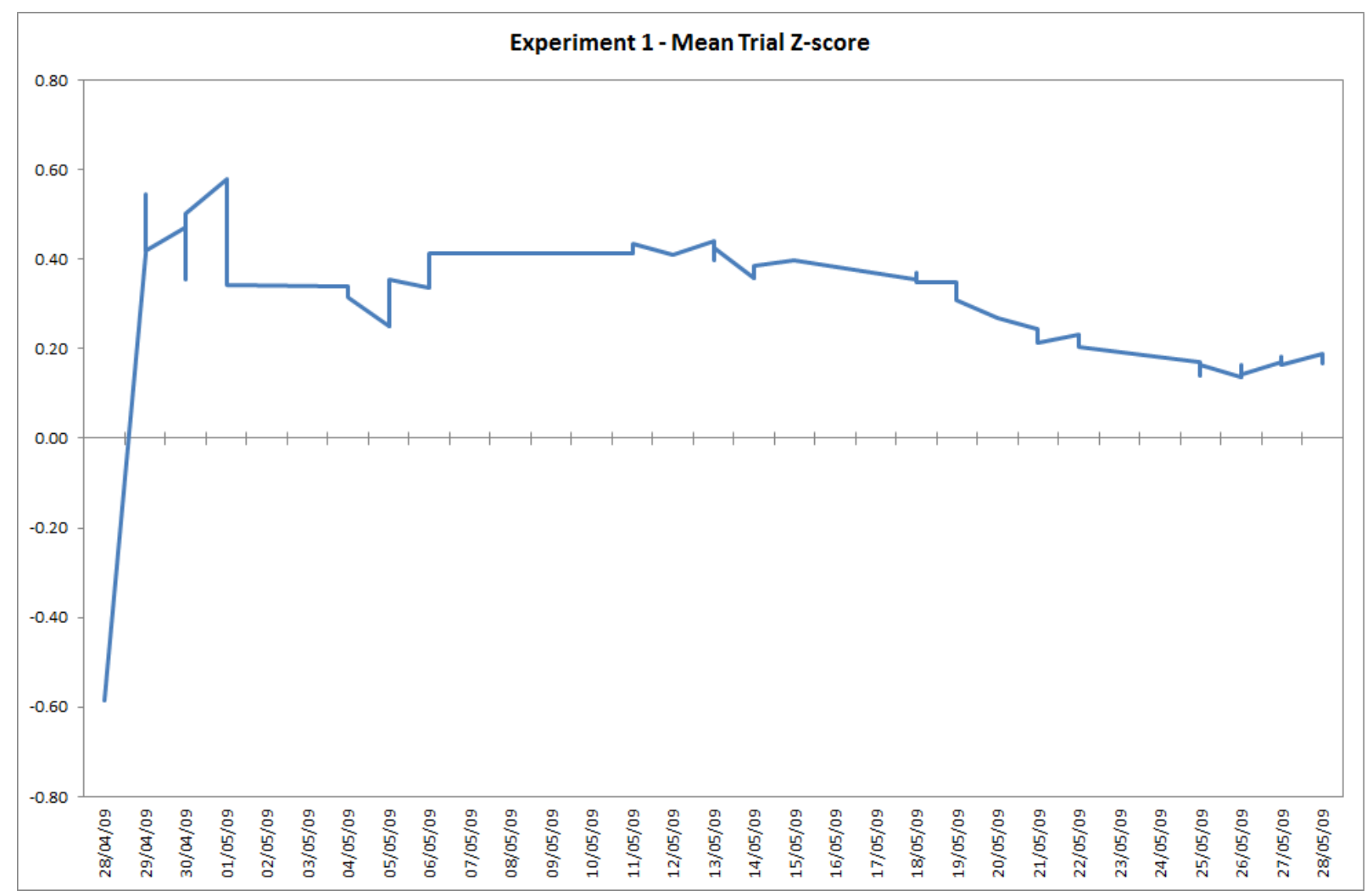

Fig. (1). The change in the mean Z-score with the time of testing in Experiment 1. 
motivation to get the prize inflated participants' level of anxiety, and this eliminated the ESP effect. In contrast, under the normal level of anxiety (NER condition), participants who have never participated in the remote viewing testing were able to show a modest but significant ESP effect.

Another way of explaining the results is through the experimenter's expectancy effect. A summary of 345 studies of eight various categories (such as reaction time, animal learning, laboratory interviews, and other) has shown that when researchers expect certain results from their subjects they unwittingly treat them in such a way as to increase the probability that the subjects respond as expected [8]. Regarding this experiment, one can assume that the experimenter expected to obtain a significant difference in performance between participants in the ER and NER conditions, and this difference was indeed obtained. However, the mismatch between the expectation (better performance in the ER condition) and the results (better performance in the NER condition) prevented the possibility of interpreting the experimenter's expectancy effect as the effect made by the experimenter on the participants, since, with all the differences between the experiments, in the "direct" experimenter's expectancy effect one feature was stable - the effect always happened in the expected direction, and not contrary to this direction [8]. However, there is still a possibility to employ the experimenter's expectancy effect for explaining the results of this experiment, by interpreting this effect as the effect of the experimenter's subconscious expectations on the process of generating the target picture (the subconscious experimenter's expectancy effect). The computer program selected the target using Excel's random number generator, which was seeded from the system timer when the "Select Target" button was pressed. It is possible that the experimenter subconsciously employed ESP to time the pressing of this button so as to influence the target selection. Such interactions between the mind and random target selection have been reported previously $[9,10]$; this theory has been termed "Decision Augmentation Theory".

The second interpretation is supported by the decline in the overall performance with the time of testing, which was observed in this experiment. Indeed, assuming that the experimenter's mind can directly affect the experiment's results, the experiment's results could be influenced by a number of psychological and physiological factors, such as experimenter's fatigue, drop in interest and motivation, bad mood, distraction from the experiment by extraneous circumstances, and so forth. Each of these factors, or their combination, might have made the subconscious experimenter effect decline with the time of testing, both within one experiment, and in subsequent replications of this experiment.

In order to examine which of the two alternative explanations (participants' anxiety level explanation, or experimenter's subconscious expectancy explanation) is more likely, in Experiment 2 of this study the difference between ER and NER condition was made less marked, by decreasing the extra reward for successful attempts by 10 times, from $£ 80$ to $£ 8$. If the participants' increased anxiety level was the factor behind a significant drop in results in the
ER condition of Experiment 1, then in Experiment 2 participants $\mathrm{Z}$ score in the ER condition should significantly increase, due to the decrease in their anxiety level. Indeed, expecting an extra amount of $£ 8$ for a success is a far less motivating factor than expecting an extra $£ 80$ to gain, and this, according to the "optimum of motivation" law, should decrease participants' anxiety level and, respectively, increase their ESP performance in the ER condition, without decreasing the performance in the standard NER condition at the same time.

If, however, it was the subconscious experimenter's expectancy effect that made the results of Experiment 1 to be so markedly different in the two conditions of Experiment 1, then in Experiment 2 the difference between the results of the two conditions should become less significant, due to the difference between the conditions becoming 10 times smaller. In addition, the overall performance in Experiment 2 should be expected to be significantly less impressive than it was in Experiment 1, due to the experimenter's fatigue and habituation to the experimental procedure. Indeed, while consciously the experimenter would still be doing his best at conducting the experiment and rating the photographs, the effort would become routine and less inspiring, and this might negatively affect the results.

\section{EXPERIMENT 2}

\section{Method}

\section{Participants}

Participants were 50 graduates and undergraduates at Lancaster University, UK, 22 males and 28 females, age range 18 to $31, \mathrm{M}=22.4, \mathrm{SD}=2.53$. None of the participants had taken part in any psi research before. Each participant completed one trial and the study comprised a total of 50 trials. Twenty-five participants were involved in each of the 2 conditions: extra reward (ER) and no extra reward (NER).

\section{Materials}

Materials were the same as in Experiment 1, except that the $\mathrm{S} \& \mathrm{G}$ scale was not used.

\section{Procedure}

Procedure was the same as in Experiment 1, with 2 exceptions: the $S \& G$ scale was not used, and the extra reward in the ER condition was $£ 8$ for a successful attempt.

\section{Design}

The independent variable was condition (2: ER versus NER), and the dependent variable was $\mathrm{Z}$ score.

\section{Expectations}

We expected that if the participants' increased anxiety level was the factor behind a significant drop in results in the ER condition of Experiment 1, then in Experiment 2 participants' mean Z score in the ER condition should significantly increase, due to the decrease in their anxiety 
level, whereas the performance in the NER condition would be about the same as in Experiment 1.

If, however, it was the subconscious experimenter's expectancy effect that shaped the pattern of results of Experiment 1, then in Experiment 2 the difference between the results of the two conditions should become less marked, due to the difference between the conditions becoming 10 times smaller. In addition, the overall performance in Experiment 2 should be expected to be a significantly less impressive than it was in Experiment 1, due to the experimenter's fatigue and habituation to the procedure.

\section{Results}

In the NER condition, mean $\mathrm{Z}=-0.15, \mathrm{SD}=0.82$. Onesamplet-test indicated that in this condition the deviation of mean $\mathrm{Z}$ from zero is not significant, with $\mathrm{t}=-0.91, \mathrm{p}=0.373$ (two tailed). In the ER condition, mean $\mathrm{Z}=-0.28, \mathrm{SD}=0.85$. According to one-samplet-test, in this condition mean $\mathrm{Z}$ was not significantly different from zero, with $\mathrm{t}=-1.65$, $\mathrm{p}=0.112$ (two tailed).

A one-way ANOVA for condition indicated no main effect for condition, $\mathrm{F}(1,48)=0.32, \mathrm{p}=0.57$ (two tailed), $\eta 2$ $=0.007$.

In the first half of testing (25 trials), mean $Z=-0.09$, $\mathrm{SD}=0.94$, and in the second (25 trials) mean $\mathrm{Z}=-0.33$, $\mathrm{SD}=0.83$. A one-way ANOVA for time of testing (2) showed no significant difference, $\mathrm{F}(1,48)=0.88, \mathrm{p}=0.35$, $\eta 2=0.02$.
A two-way ANOVA for experiment (2) and condition (2) indicated a main effect for experiment, $F(1,96)=5.24$, $\mathrm{p}=0.02, \eta 2=0.05$, with Experiment 1 having a significantly higher mean $\mathrm{Z}$ score than Experiment 2. A marginally significant effect was also obtained for condition, $\mathrm{F}(1,96)=3.61, \mathrm{p}=0.06, \eta 2=0.04$. There was no significant interaction between experiment and condition factors.

\section{DISCUSSION}

The results of Experiment 2 favour the subconscious experimenter expectancy explanation of the Experiment 1 results, and not the participants' level of anxiety explanation. Indeed, in spite of the decrease of the extra reward in the ER condition of Experiment 2 by 10 times, the results in the ER condition of Experiment 2 did not significantly increase, as the participants' anxiety explanation predicted, and remained not significantly different from zero. To the contrary, the predictions made by the subconscious experimenter expectancy effect explanation of Experiment 1 results came true: the difference between the results of ER and NER conditions in Experiment 2 was not significant, and overall performance in Experiment 2 was significantly below that in Experiment 1.

Interestingly, even though the overall performance in Experiment 2 did not significantly decrease in the second 25 trials, the decline in performance with time of testing is, nevertheless, visible on the graph, which shows the dynamics of the mean $\mathrm{Z}$ scores across testing (see Fig. 2).

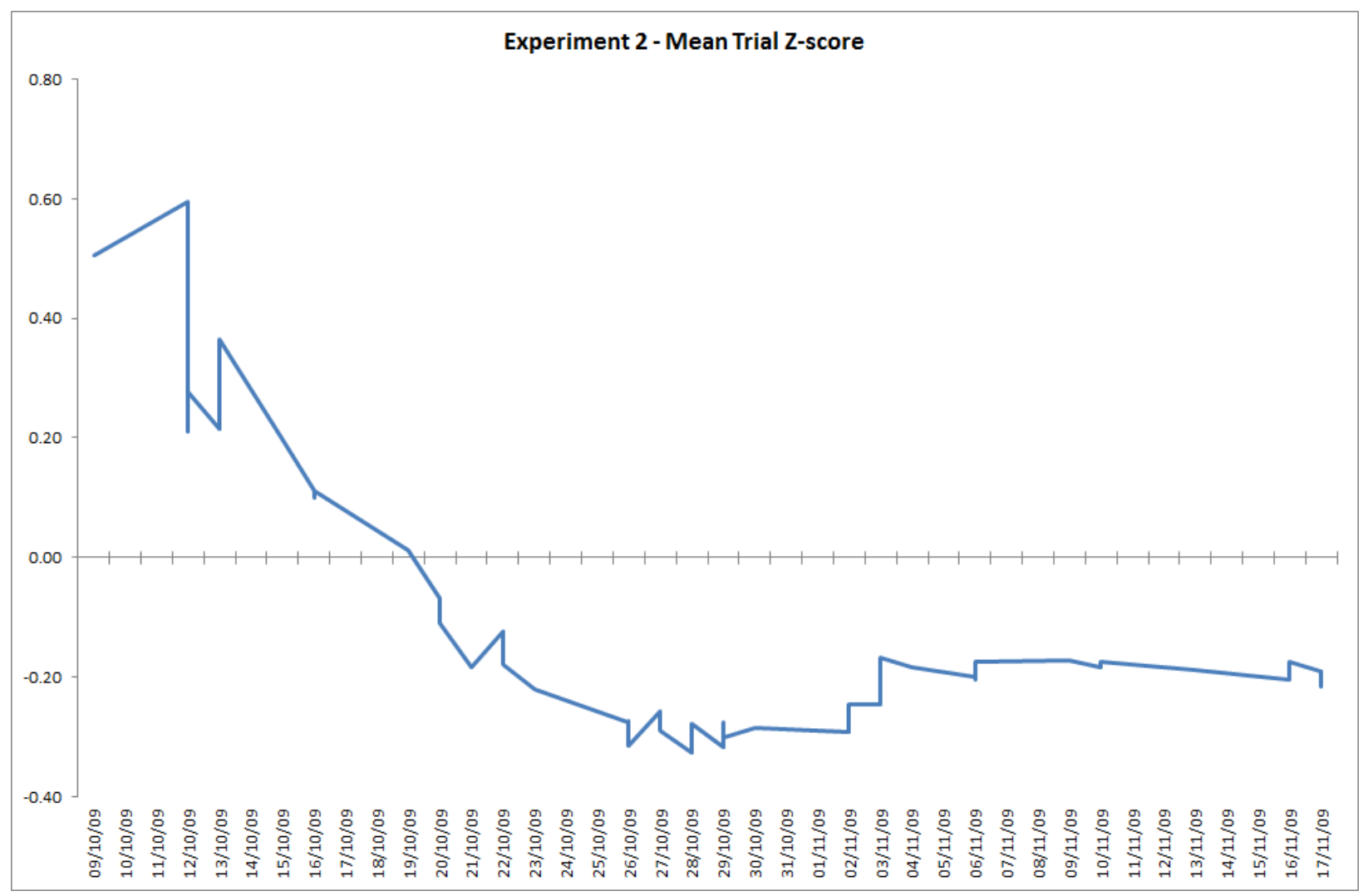

Fig. (2). The change in the mean Z-score with the time of testing in Experiment 2. 
This decline is an additional indication that the experimenter's mind was affecting the results in Experiments 2, by producing a drop in results. As both overall mean $\mathrm{Z}$ score in the first and second halves of testing were not significantly different from zero, the difference between these mean scores was also not significant.

\section{GENERAL DISCUSSION}

In Experiment 1, the hypothesis was tested that the ESP performance on the Ed May's task improves with the magnitude of material reward for success. The results of this experiment indicated that, contrary to the expectation, participants in the NER condition scored positively and significantly on the ESP task, whereas participants in the ER condition, who received an extra amount of $£ 80$ for a successful attempt, scored near zero.

Two explanations of this result were offered. According to one explanation, participants' poor performance in the ER condition was due to their increased anxiety about the results of their attempt. This explanation is based on the "law of optimum of motivation", which contends that highly motivated participants perform poorly due to the high level of anxiety. According to the other explanation, the results were shaped by the subconscious experimenter's expectancy effect. The experimenter expected the results in two conditions to be different and, subconsciously, affected the random target selection in one condition, but not in the other condition. The second explanation was supported by the significant reduction of overall performance in the second half of testing in Experiment 1, presumably due to the experimenter's fatigue.

The results of Experiment 2 supported the "subconscious experimenter's expectancy" explanation, and not the "participants' anxiety" explanation. In that experiment, the magnitude of the material reward for successful attempts was decreased by 10 times (from $£ 80$ in Experiment 1 to $£ 8$ in Experiment 2), with the aim to reduce the participants' anxiety and improve their performance in the ER condition. Instead, in Experiment 2 participants in both conditions scored near zero. This result is in favour of the experimenter's expectancy explanation of Experiment 1 results: in Experiment 2, due to the experimenter's fatigue and habituation to the procedure, the experimenter's subconscious influence on the random target selection was terminated, and this resulted in chance performance in both conditions.

One possible objection to this interpretation is that the experimenter's fatigue did indeed affect the results of Experiment 2, but not due to a cessation of the experimenter's influence on the random target selection, but because the experimenter's effort to maintain the flow of experiment and rating the pictures in Experiment 2 were at a lower level than in Experiment 1. This explanation is, however, unlikely. In this study, the experimental procedure makes high demand on both participants' and the experimenter's effort and time. Because of this, for the experimenter relaxing the effort in Experiment 2 would mean wasting a month of the everyday effort, plus a substantial amount of participants' money. Rather, it is more likely that consciously, in Experiment 2 the experimenter was functioning as effectively as in Experiment1, but subconsciously, his mind ceased its influence on the random target selection process and allowed it to perform as it supposed to, by picking up the target picture at the level of chance.

But if the experimenter's mind affected the random target selection and thus shaped the experiments' results, the question arises of how exactly this influence operated. There are two possible ways that this effect could go. One way is increasing the probability of the hits, by making the computer pick those pictures that the experimenter rated as most likely to be the target picture with a frequency significantly exceeding chance. Another is to decrease the number of misses, i.e., to block the computer from picking the pictures that the experimenter rated as the least likely to be the target pictures and thus shifting the balance in favour of hits.

While the overall parametric statistics does not allow answering this question, looking at results more closely can suggest the answer. Indeed, in Experiment 1, in the NER condition hits (when the target object was marked by the experimenter as the most probable, producing $\mathrm{Z}=0.9$ or larger), happened 7 times, whereas misses (when the target object was marked as the least probable, producing $Z=-0.9$ or smaller) happened just once. To the contrary, in the ER conditionthere were 6 hits and 6 misses. The number of hits in both conditions is approximately equal, but the number of misses in the ER condition is significantly larger than in the NER condition, Mann-Whitney $U=375, \mathrm{p}=0.044$ (twotailed). This shows that in the NER condition the number of hits was at the expected chance level (i.e., $24 \%$ of the total number of trials, with the chance level predicting $20 \%$ of hits), but the number of misses was significantly below chance (i.e., 4\%, with the chance level predicting 20\%), whereas in the ER condition both the numbers of hits and misses were at chance level. Furthermore, 6 out 7 misses happened in the second half of testing. This suggests, that in the first half of testing there was no difference between the ER and NER conditions' results, but in the second half of testing some factor altered the proportion between hits and misses in the ER condition, allowing more misses to happen and thus reducing the overall result in this condition down to chance, but this did not happen in the NER condition. It is possible to assume that this factor was a combination of the experimenter's expectancy (that results in the two conditions should be different) and fatigue: due to the fatigue and habituation, the experimenter's mind stopped blocking misses from happening, but this relaxation of "misses inhibition" was one-sided and affected only the ER condition.

In contrast, in Experiment 2, in which no substantial difference was expected between the results of the two conditions, numbers of hits and misses within conditions were approximately equal (4 hits and 6 misses in the NER condition, and 4 hits and 9 misses in the ER condition), and not different from chance. This suggests that in Experiment 2 , in both conditions the experimenter's mind had no effect on the random target selection process. The overall lower performance in Experiment 2, compared with that in Experiment 1, adds to the suggestion that in both 
experiments, the combination of experimenter's expectancy and fatigue (habituation) determined the patterns of results.

The effect that remains unexplained is why the experimenter's subconscious expectancy effect worked at such low level of precision. Specifically, the expectation that the results between conditions of Experiments 1 and 2 would or would not be different has worked, but the expectation of the direction in which the results between conditions of Experiment 1 would differ did not. It is possible to assume that all the experimenter's subconscious expectancy can do is disturb the chance performance of the RNG, by diminishing the probability of misses in one of the two contrasting conditions, but determining in what condition exactly such "error inhibition" should happen exceeds the power of the subconscious expectancy effect.

\section{REFERENCES}

[1] Stanford RG, Rust, P. Psi-mediated helping behavior: Experimental paradigm and initial results. In: Morris JD, Roll WG, Morris RL, Eds. Res Parapsy, Scarecrow, Metuchen NJ; 1976; pp. 109-10.
[2] Batson CD. How social an animal? The human capacity for caring. Amer Psychol 1990; 45: 336-346.

[3] Eysenck HJ, Sargent C. Explaining the unexplained. Mysteries of the paranormal. BCA: London - England: Prion 1993; pp.123-124.

[4] Subbotsky E, Ryan A. Motivation, belief and geomagnetic activity in a remote viewing task. The $33^{\text {rd }}$ International Conference of the Society for Psychical Research, Nottingham; United Kingdom. 2009.

[5] May EC, Faith LV, Blackman M, Bourgeois B, Kerr N, Woods L. A target pool and database for anomalous cognition experiments: Proceedings of the $40^{\text {th }}$ Annual Convention of the Parapsychological Association; 1999

[6] Thalbourne MA, Dean PS. A new instrument for measuring the sheep-goat variable: its psychometric properties and factor structure. J Soc Psych Res1993; 59: 172-86.

[7] Dictionary of Psychology. Definition of Yerkes-Dodson Law. Information retrieved on $12^{\text {th }}$ October 2008 at the official website: http://www.encyclopedia.com/doc/1O87-YerkesDodsonlaw.html

[8] Rosenthal R, Rubin DB. Interpersonal expectancy effects: the first 345 studies. Behav Br Sci 1978; 3: 377-415.

[9] May EC, Utts JM, Spottiswoode SJP. Decision augmentation theory: Applications to the random number generator database. J Sci Explor 1995; 9: 453-88.

[10] May EC, Utts JM, Spottiswoode SJP. Decision augmentation theory: Toward a model for anomalous mental phenomena. J Parapsych 1995; 59: 195-220.

Received: February 25, 2014

Revised: May 29, 2014

Accepted: June 12, 2014

(c) Subbotsky and Ryan; Licensee Bentham Open.

This is an open access article licensed under the terms of the Creative Commons Attribution Non-Commercial License (http://creativecommons.org/licenses/ by-nc/3.0/) which permits unrestricted, non-commercial use, distribution and reproduction in any medium, provided the work is properly cited. 